\title{
Multichannel matching pursuit for seismic trace decomposition
}

\author{
Yanghua Wang ${ }^{1}$
}

\begin{abstract}
The technique of matching pursuit can adaptively decompose a seismic trace into a series of wavelets. However, the solution is not unique and is also strongly affected by data noise. Multichannel matching pursuit (MCMP), exploiting lateral coherence as a constraint, might improve the uniqueness of the solution. It extracts a constituent wavelet that has an optimal correlation coefficient to neighboring traces, instead of to a single trace only. According to linearity theory, a wavelet shared by neighboring traces is the best match to the average of multiple traces, and therefore it might effectively suppress the data noise and stabilize the performance. It is found that the MCMP scheme greatly improves spatial continuity in decomposition and can generate a plausible time-frequency spectrum with high resolution for reservoir detection.
\end{abstract}

\section{INTRODUCTION}

Matching pursuit adaptively decomposes a seismic trace into a series of constituent wavelets (Mallat and Zhang, 1993; Wang, 2007). Each of these wavelets, selected from a dictionary consisting of abundant wavelets, also called atoms, has an optimal correlation coefficient with the trace. The intention is to overcome limitations in conversional time-frequency spectrum generation methods such as the Gabor transform and the wavelet transform. Figure 1a displays a seismic trace, and Figure 1b-d shows the time-frequency spectra generated from the Gabor transform, the wavelet transform, and matching pursuit, respectively. In the Gabor transform, the size of the time window sliding along the trace is predefined and usually is a constant, tapered with a Gaussian function (Gabor, 1946). Therefore the spectrum depends on the predefined window size (Figure 1b). In the wavelet transform (Mallat, 2009), the time duration of a constituent wavelet is predefined also and is set to be inversely proportional to its dominant frequency (Figure 1c). On the contrary, matching pursuit, with a flexible wavelet size, can adaptively match the true signature in the seismic trace. The resultant time-frequency spectrum (Figure 1d) shows that each wavelet has distinct durations in the time and frequency axes.

When applying single-channel matching pursuit (SCMP) to real seismic data (Figure 2a), however, a problem exists: nonuniqueness of the decomposition. Because of this nonuniqueness, the data noise also severely affects the decomposition. As SCMP performs decomposition on each trace independently, the extracted wavelets, and the reconstructed profile (the left panel of Figure 2b), are lacking in spatial continuation between seismic traces, which results in some residuals (the right panel of Figure 2b). Consequently, the time-frequency spectrum will lack lateral continuation along a profile.

In this study, matching pursuit is implemented in a multichannel fashion so as to explore lateral continuity of seismic events and meanwhile to suppress the noise effect in decomposition (Durka et al., 2005; Studer et al., 2006). In this way, multichannel matching pursuit (MCMP) might partially overcome the problem of nonuniqueness. With the constraint of lateral coherency, extracted wavelets and the time-frequency spectrum will have optimal lateral continuation along the profile. Such constrained decomposition also stabilizes the convergence, and consequently, the reconstructed profile can accurately resemble the original seismic section (Figure 2c).

In the following sections, first I summarize the basics of the MCMP algorithm, the core of which is that a constituent wavelet should be shared by neighboring traces. Then I present a robust implementation of MCMP based on linearity theory. This linearity also leads to discussion on the stability of performance. Finally, I demonstrate the application of MCMP with two examples: The first is to remove a strong coal-seam reflection by exploiting the spatial continuity, and the other is to generate plausible time-frequency spectra for gas reservoir detection.

\section{MULTICHANNEL MATCHING PURSUIT}

Given a seismic trace $f(t)$, single-channel matching pursuit is implemented iteratively. After $n-1$ iterations, a total of $n-1$ wavelets are extracted from the trace and the residual trace is $R^{(n-1)}\{f(t)\}$,

Manuscript received by the Editor 31 October 2009; revised manuscript received 28 January 2010; published online 13 August 2010.

${ }^{1}$ Imperial College London, Department of Earth Science and Engineering, Centre for Reservoir Geophysics, London, U. K. E-mail: yanghua.wang@ imperial.ac.uk.

๑) 2010 Society of Exploration Geophysicists. All rights reserved. 


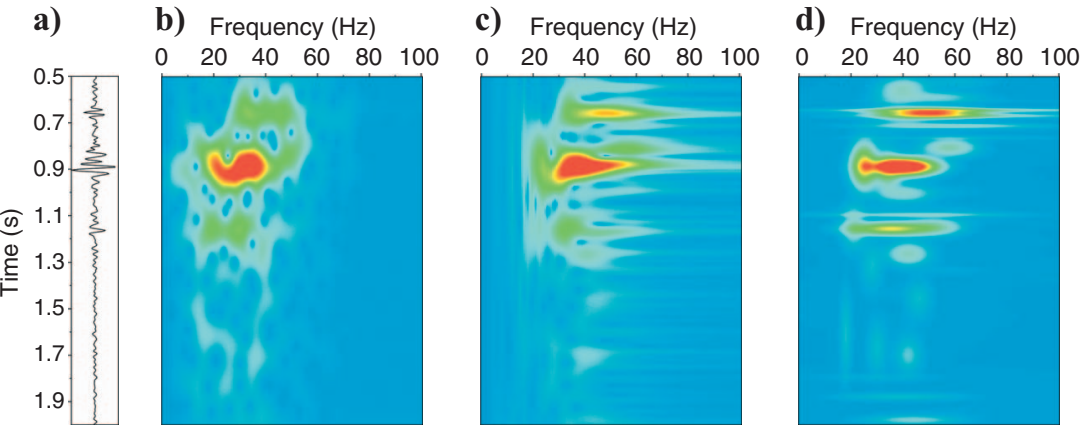

Figure 1. (a) A single seismic trace. (b) Time-frequency spectrum obtained by the Gabor transform. (c) The spectrum generated from the wavelet transform. (d) The spectrum generated from matching pursuit.
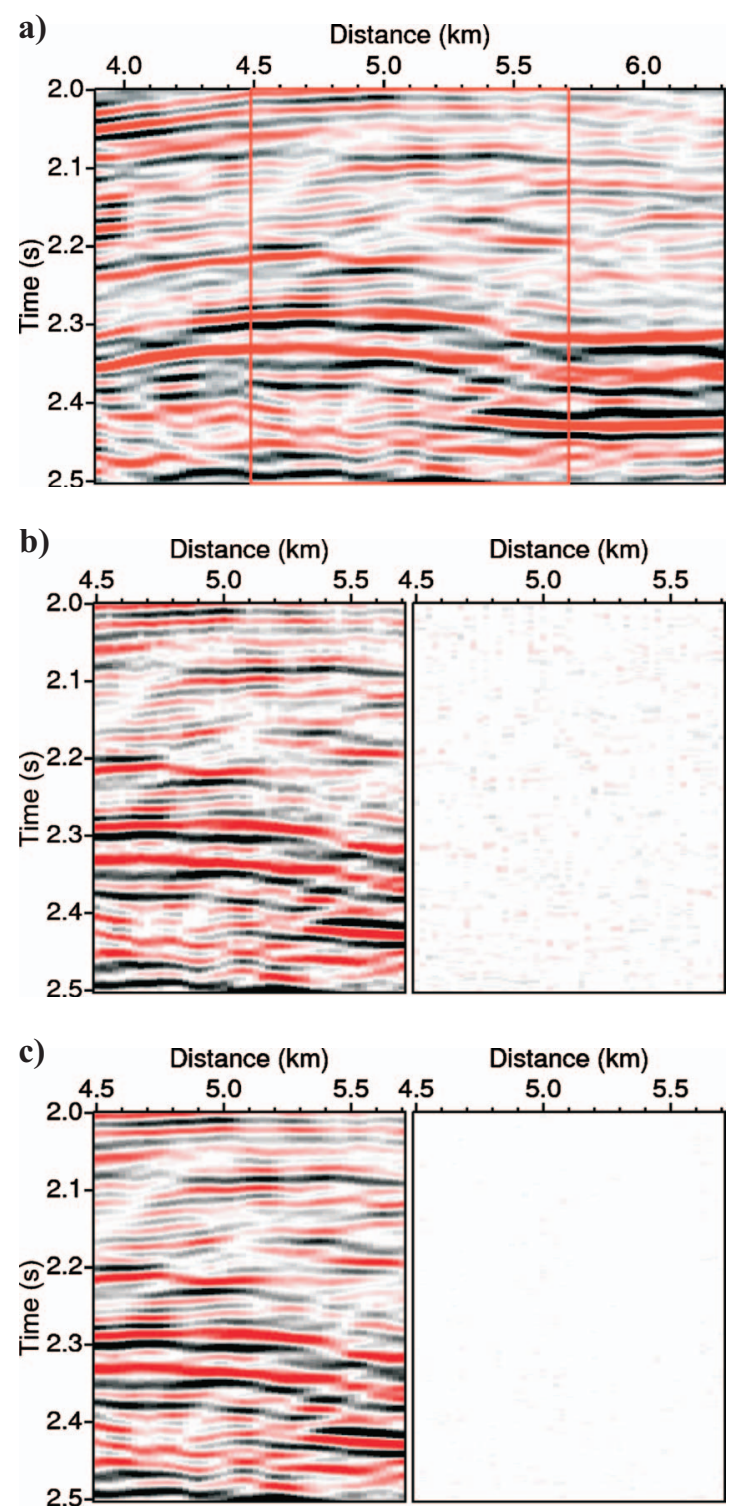

Figure 2. (a) A sample seismic profile for matching pursuit (red frame indicates the zoom-in area shown in b and c). (b) Reconstructed profile by single-channel matching pursuit and the residuals. (c) Reconstructed profile using multichannel matching pursuit and the residuals. This demonstrates the completeness of multichannel decomposition. where $R$ is a linear operator, called the residuum operator, and $R^{(0)}\{f(t)\}=f(t)$. At the $n$th iteration, the extracted wavelet is $w_{n}(t)$, and the residual trace is

$$
R^{(n)}\{f(t)\}=R^{(n-1)}\{f(t)\}-w_{n}(t) .
$$

The wavelet $w_{n}(t)$ is presented as

$$
w_{n}(t)=a_{n} g_{\gamma_{n}}(t)
$$

where $g_{\gamma_{n}}(t)$ is a basic wavelet, or an atom, obtained after the $n$th iteration, which has the optimal correlation coefficient with the residual trace $R^{(n-1)}\{f(t)\} ; \gamma_{n}$ is a group of parameters representing the atom; and $a_{n}$ is the amplitude scalar.

For multichannel matching pursuit, assuming there is a group of neighboring traces $\left\{f_{1}(t), f_{2}(t), \ldots, f_{L}(t)\right\}$ around a single trace $f_{\ell}(t)$, the single trace $f_{\ell}(t)$ is decomposed into a series of wavelets based on this group of traces. As defined in equation 1 , the residual trace at the beginning is $R^{(0)}\left\{f_{\ell}(t)\right\}=f_{\ell}(t)$, and at the $n$th iteration is

$$
R^{(n)}\left\{f_{\ell}(t)\right\}=R^{(n-1)}\left\{f_{\ell}(t)\right\}-a_{\ell, n} g_{\gamma_{n}}(t)
$$

where $g_{\gamma_{n}}(t)$ is an atom extracted from the $n$th iteration. Note here that, although $a_{\ell, n}$ is the amplitude of the wavelet $w_{\ell, n}$ for this individual trace $\ell, g_{\gamma_{n}}$ is an atom shared by all $L$ traces within the group. After $N$ iterations, we might decompose the single trace $f_{\ell}(t)$ into $N$ wavelets as

$$
f_{\ell}(t)=\sum_{n=1}^{N} a_{\ell, n} g_{\gamma_{n}}(t)+R^{(N)}\left\{f_{\ell}(t)\right\}
$$

where $R^{(N)}\left\{f_{\ell}(t)\right\}$ is the final residual trace.

The Morlet wavelet (Morlet et al., 1982a, 1982b) is used as the atom in matching pursuit decomposition for seismic traces. A basic Morlet wavelet $m(t)$ centered at the abscissa $t=u$ is defined as

$$
m(t)=\exp \left[-\left(\frac{\ln 2}{\pi^{2}}\right) \frac{\omega_{m}^{2}(t-u)^{2}}{\sigma^{2}}\right] \exp \left[i\left(\omega_{m}(t-u)+\phi\right)\right]
$$

where $\omega_{m}$ is the mean angular frequency, $\phi$ is the phase, and $\sigma$ is a constant value controlling the wavelet width. The Morlet wavelet has a constant shape ratio, diameter/mean period = constant, where the diameter or duration is measured at half of the maximum am-plitude of the wavelet envelope, or $-6 \mathrm{~dB}$ in logarithmic scale. Therefore, there are four parameters $\gamma_{n}=\left\{u_{n}, \sigma_{n}, \omega_{n}, \phi_{n}\right\}$ presenting an atom $g_{\gamma_{n}}(t)$ : the time abscissa $u_{n}$, the scale $\sigma_{n}$, the central frequency $\omega_{n} \equiv \omega_{m, n}$, and the phase $\phi_{n}$.

The use of Morlet wavelets as constituent atoms in a matching pursuit is based on the following effectiveness and efficiency considerations (Wang, 2007). First, the Morlet wavelet can represent the attenuation behavior of wave propagation (Morlet et al., 1982a, b). Second, using the Morlet wavelet as the atom, instead of searching within a vast wavelet dictionary, saves wavelet-searching time. Third, by using an analytic form, analytic expressions can be derived for the computation of wavelet decomposition and time-frequency spectrum generation, as shown in the following sections. 


\section{IMPLEMENTATION AND LINEARITY}

Within each iteration, the implementation can be divided into three steps. The first step is a single-channel matching pursuit to generate an initial estimate of the atom from an average residual trace. The second step is to refine the atom in a multichannel fashion. The third step is to estimate the amplitude of the wavelet corresponding to each trace.

In the first step, averaging $L$ residual traces within the group generates a single residual trace

$$
R^{(n-1)}\{y(t)\}=\frac{1}{L} \sum_{\ell=1}^{L} R^{(n-1)}\left\{f_{\ell}(t)\right\},
$$

where $R^{(0)}\{y(t)\}=\frac{1}{L} \sum_{\ell} f_{\ell}(t)$. Then, performing a Hilbert transform on $R^{(n-1)}\{y(t)\}$, we can find the instantaneous frequency $\omega_{n}$ and the instantaneous phase $\phi_{n}$, corresponding to the maximum of the instantaneous envelope. The corresponding time is the abscissa $u_{n}$. With fixed $u_{n}, \omega_{n}$, and $\phi_{n}$ values, we search for an optimal parameter $\sigma_{n}$ using the following equation:

$$
g_{\gamma_{n}}(t)=\arg \max _{g_{\gamma_{n}} \in D} \frac{\left\langle R^{(n-1)}\{y(t)\}, g_{\gamma_{n}}(t)\right\rangle}{\left\|g_{\gamma_{n}}(t)\right\|},
$$

where $D=\left\{g_{\gamma_{n}}(t)\right\}_{\gamma_{n} \in \Gamma}$ is a comprehensive dictionary of the constituent wavelets, $\left\langle R^{(n-1)}\{y(t)\}, g_{\gamma_{n}}(t)\right\rangle$ denotes the inner product of the seismic trace residual $R^{(n-1)}\{y(t)\}$ with atom $g_{\gamma_{n}}(t)$, and $\left\|g_{\gamma_{n}}(t)\right\|$ $=\sqrt{\left\langle g_{\gamma_{n}}(t), g_{\gamma_{n}}(t)\right\rangle}$ normalizes the atom $g_{\gamma_{n}}(t)$. For the Morlet wavelet, an analytic expression for $\left\|g_{\gamma_{n}}(t)\right\|$ is given by Wang (2007):

$$
\left\|g_{\gamma_{n}}\right\|^{2}=\frac{\pi}{2} \sqrt{\frac{\pi}{2 \ln 2}} \frac{\sigma_{n}}{\omega_{n}}\left(1+\exp \left[-\frac{\pi^{2} \sigma_{n}^{2}}{2 \ln 2}\right] \cos \phi\right) .
$$

Considering $\sigma_{n}$ as a variable is a powerful feature of the matching pursuit process.

In the second step, we refine the parameters $\gamma_{n}=\left\{u_{n}, \sigma_{n}, \omega_{n}, \phi_{n}\right\}$ over a group of preselected, uniformly distributed values by maximizing the sum of the correlation coefficients in each residual trace:

$$
g_{\gamma_{n}}(t)=\arg \max _{g_{\gamma_{n}} \in D} \frac{\sum_{\ell=1}^{L}\left|\left\langle R^{(n-1)}\left\{f_{\ell}(t)\right\}, g_{\gamma_{n}}(t)\right\rangle\right|}{\left\|g_{\gamma_{n}}(t)\right\|} .
$$

Thus, the atom $g_{\gamma_{n}}(t)$ is the best fit to all traces within a group.

In the third step, we estimate the amplitude $a_{\ell, n}$ corresponding to each individual trace $\ell$ by

$$
a_{\ell, n}=\frac{\left|\left\langle R^{(n-1)}\left\{f_{\ell}(t)\right\}, g_{\gamma_{n}}(t)\right\rangle\right|}{\left\|g_{\gamma_{n}}(t)\right\|^{2}}
$$

and finally the matched wavelet is found as $w_{\ell, n}(t)=a_{\ell, n} g_{\gamma_{n}}(t)$.

These three steps are performed iteratively for $n=1,2, \ldots, N$. The procedure terminates when the residual energy is less than a preset threshold or the number of iterations reaches a preset maximum value.

The robust multichannel implementation scheme described above exploits the linearity of the residuum operator $R^{(n)}$ (Durka et al., 2005). Initially, because $R^{(0)}$ is an identity operator, $R^{(0)}\{y(t)\}=y(t)$, we have

$$
\frac{1}{L} \sum_{\ell=1}^{L} R^{(0)}\left\{f_{\ell}(t)\right\}=R^{(0)}\left\{\frac{1}{L} \sum_{\ell=1}^{L} f_{\ell}(t)\right\} .
$$

For the first iteration, the residuum operator $R^{(1)}$ is a linear operator so that

$$
\begin{aligned}
\frac{1}{L} \sum_{\ell=1}^{L} R^{(1)}\left\{f_{\ell}(t)\right\} & =\frac{1}{L} \sum_{\ell=1}^{L}\left[R^{(0)}\left\{f_{\ell}(t)\right\}-a_{\ell, 0} g_{\gamma_{0}}(t)\right] \\
& =R^{(0)}\left\{\frac{1}{L} \sum_{\ell=1}^{L} f_{\ell}(t)\right\}-\frac{1}{L} \sum_{\ell=1}^{L} a_{\ell, 0} g_{\gamma_{0}}(t) \\
& =R^{(1)}\left\{\frac{1}{L} \sum_{\ell=1}^{L} f_{\ell}(t)\right\}
\end{aligned}
$$

where $\frac{1}{L} \sum_{\ell} a_{\ell, 0} g_{\gamma_{0}}(t)$ is an average wavelet. By induction, for the $n$th iteration, $R^{(n)}$ is a linear operator, so

$$
\begin{aligned}
\frac{1}{L} \sum_{\ell=1}^{L} R^{(n)}\left\{f_{\ell}(t)\right\} & =\frac{1}{L} \sum_{\ell=1}^{L}\left[R^{(n-1)}\left\{f_{\ell}(t)\right\}-a_{\ell, n} g_{\gamma_{n}}(t)\right] \\
& =R^{(n-1)}\left\{\frac{1}{L} \sum_{\ell=1}^{L} f_{\ell}(t)\right\}-\frac{1}{L} \sum_{\ell=1}^{L} a_{\ell, n} g_{\gamma_{n}}(t) \\
& =R^{(n)}\left\{\frac{1}{L} \sum_{\ell=1}^{L} f_{\ell}(t)\right\} .
\end{aligned}
$$

This means that the sum of residual traces, $\Sigma_{\ell} R^{(n)}\left\{f_{\ell}(t)\right\}$, is equal to the residual of the trace summation, $R^{(n)}\left\{\sum_{\ell} f_{\ell}(t)\right\}$.

This linearity analysis also leads to

$$
\begin{aligned}
\frac{1}{L} \sum_{\ell=1}^{L} & \left\langle R^{(n-1)}\left\{f_{\ell}(t)\right\}, g_{\gamma_{n}}(t)\right\rangle \\
& =\left\langle\frac{1}{L} \sum_{\ell=1}^{L} R^{(n-1)}\left\{f_{\ell}(t)\right\}, g_{\gamma_{n}}(t)\right\rangle \\
& =\left\langle R^{(n-1)}\left\{\frac{1}{L} \sum_{\ell=1}^{L} f_{\ell}(t)\right\}, g_{\gamma_{n}}(t)\right\rangle,
\end{aligned}
$$

where the first equality is derived from the linearity of the product operator $\langle\cdot, \cdot\rangle$, and the second equality is derived from the linearity of the residuum operator. Equation 14 indicates that the sum of products across all traces is equal to the product of the sum and the atom.

In equation 9, the atom $g_{\gamma_{n}}(t)$ is shared by all traces within a group. According to equation 14, it is the best fit to the average of residual traces or the residual of trace averages. Averaging over multiple traces might effectively suppress data noise and thus stabilize the process.

\section{APPLICATION EXAMPLES}

\section{Extracting a coal-seam reflection by exploiting spatial continuity}

The first application example is to extract a strong coal-seam reflection so that the remaining target reflections will be visible from 
the seismic profile. As shown in Figure 3a, the strong reflection is about $1.5 \mathrm{~s}$ across the profile, and the target gas reservoir formation is immediately above it, between 1.4 and $1.5 \mathrm{~s}$.

To track the coherent event, we use the instantaneous phase information (Figure $3 \mathrm{~b}$ ) as the input to calculate the crosscorrelation between traces. As the phase section shows a clear continuation following the horizon at a time of about $1.5 \mathrm{~s}$, performing crosscorrelation between phase traces can automatically track the horizon. This correlation provides us the initial time abscissa $u_{\ell}$, where $\ell$ is the

Figure $3 \mathrm{c}$ displays the wavelets extracted along the horizon by using the multichannel matching pursuit process. In this application, the number of traces within a spatial window is set to be either three or five. The results are similar.

Figure $3 \mathrm{~d}$ is the same section as Figure $3 \mathrm{a}$ but after the removal of the strong coal-seam reflection. This means that it is the difference between Figure $3 \mathrm{a}$ and c. This section clearly shows weak reflections across the profile at times between 1.4 and $1.5 \mathrm{~s}$.

\section{Analyzing time-frequency distribution for gas detection}

The second application example is to generate a time-frequency spectrum for gas reservoir detection. Low-frequency shadows in the time-frequency spectrum are a direct indication for gas reservoir detection (Chakraborty and Okaya, 1995; Castagna et al., 2003; Korneev et al., 2004; Sinha et al., 2005).

After decomposing a signal $f_{\ell}(t)$ into a series of wavelets $g_{\gamma_{n}}(t)$, for $n=1,2, \ldots N$, the Wigner distribution can be used to present the amplitude distribution in the time-frequency space as trace reference.

$$
A\left\{f_{\ell}(t, \omega)\right\}=\sum_{n=1}^{N} \frac{a_{\ell, n}}{\left\|g_{\gamma_{n}}\right\|} \sqrt{W\left\{g_{\gamma_{n}}(t, \omega)\right\}},
$$

where $W\left\{g_{\gamma_{n}}(t, \omega)\right\}$ is the Wigner distribution of a selected wavelet $g_{\gamma_{n}}(t)$ and is given by

$$
\begin{aligned}
W\left\{g_{\gamma_{n}}(t, \omega)\right\}= & \frac{1}{2 \pi} \int_{-\infty}^{+\infty} g_{\gamma_{n}}\left(t+\frac{\tau}{2}\right) \bar{g}_{\gamma_{n}}\left(t-\frac{\tau}{2}\right) \\
& \times \exp [-i \omega \tau] d \tau,
\end{aligned}
$$

and $\bar{g}_{\gamma_{n}}(t)$ is the complex conjugate of $g_{\gamma_{n}}(t)$. For the Morlet wavelet $m(t)$ defined in equation 5, an analytic expression for the time-frequency spectrum can be derived as (Wang, 2007)

$$
\begin{aligned}
A\left\{f_{\ell}(t, \omega)\right\}= & \sum_{n=1}^{N} \frac{a_{\ell, n}}{\left\|g_{\gamma_{n}}\right\|}\left(\sqrt{\frac{\pi}{2 \ln 2}} \frac{\sigma_{n}}{\omega_{n}}\right)^{1 / 2} \\
& \times \exp \left[-\left(\frac{\pi^{2}}{4 \ln 2}\right) \frac{\sigma_{n}^{2}\left(\omega-\omega_{n}\right)^{2}}{\omega_{n}^{2}}\right] \\
& \times \exp \left[-\left(\frac{\ln 2}{\pi^{2}}\right) \frac{\omega_{n}^{2}\left(t-u_{n}\right)^{2}}{\sigma_{n}^{2}}\right],
\end{aligned}
$$

where $\left\|g_{\gamma_{n}}(t)\right\|$ can be estimated efficiently using the analytic expression in equation 8 .

Figure 4a shows a crooked line across six wells, and Figure 4b-d displays three frequency profiles obtained by using the multichannel matching pursuit method. We can see that, below the target gas reservoirs (yellow circles), the low-frequency shad- a)

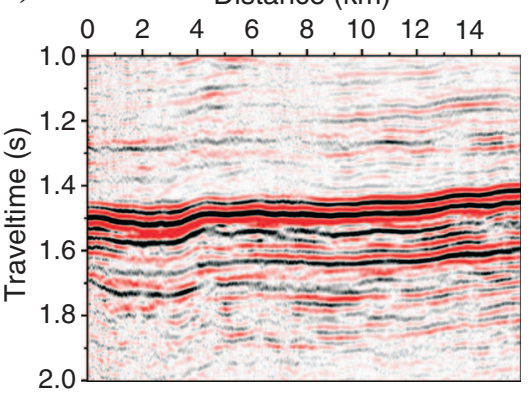

c)

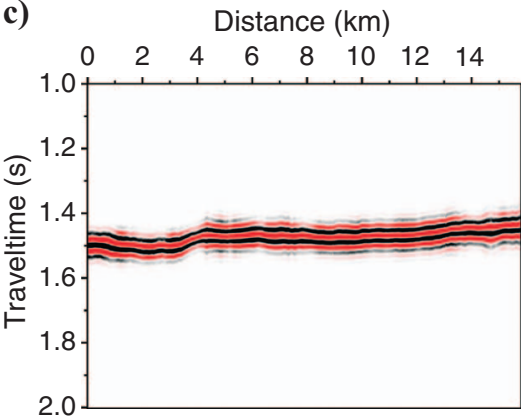

b)

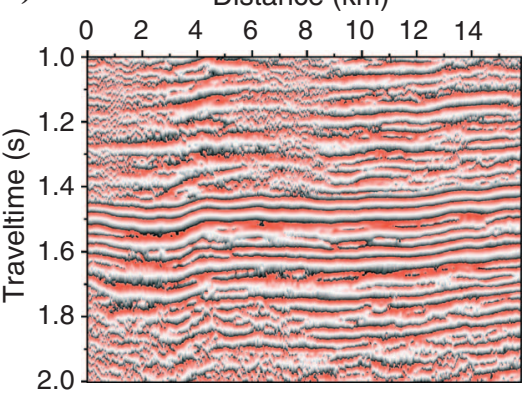

d)

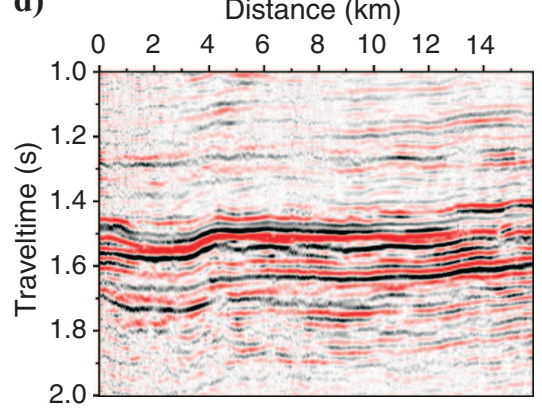

Figure 3. (a) A seismic section extracted from a 3D seismic cube. (b) The instantaneous phase section, on which the strong coal-seam reflection (at about $1.5 \mathrm{~s}$ ) can be tracked. (c) The strong coal-seam reflection is extracted from the seismic section using multichannel matching pursuit. (d) The seismic section after removing the coal-seam reflection shows weak target reflections between 1.4 and $1.5 \mathrm{~s}$. ows at $10 \mathrm{~Hz}$ are gradually reduced, but meanwhile the target gas reservoirs show strong amplitudes in 20 and $25 \mathrm{~Hz}$.

\section{DISCUSSION ON "WAVELET"}

We first look at the difference in "wavelet" between the wavelet transform and the matching pursuit decomposition. Figure 5a gives an example profile extracted from a 3D seismic cube, and Figure $5 \mathrm{~b}$ and $\mathrm{c}$ shows two constant-frequency profiles generated, respectively, by these two methods. In the wavelet transform for a constant dominant frequency, a wavelet with fixed size is correlated with a seismic trace at each sample point along the time axis, and the resultant continuous correlation coefficient gives the spectrum with respect to time. In matching pursuit, however, the size of wavelets along the time axis is flexible and is estimated adaptively from the seismic data. Therefore, the matching pursuit method shows much higher temporal resolution than the wavelet transform does.

However, the constituent wavelet in both methods is conceptually different from the wavelet used in the convolutional modeling of a seismic trace. The latter assumes a wavelet to be constant along the time axis, at least within a certain 
a)

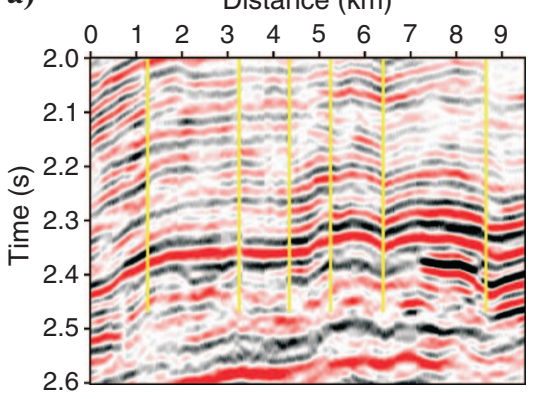

c)

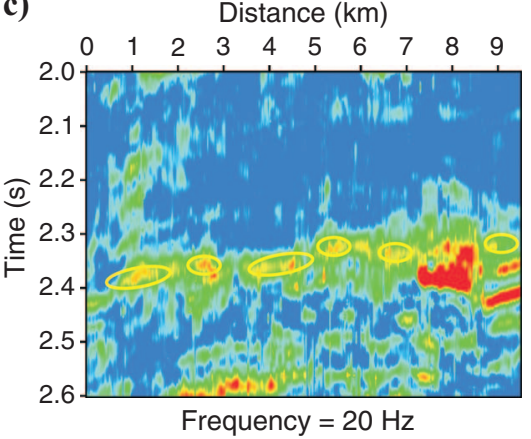

b)

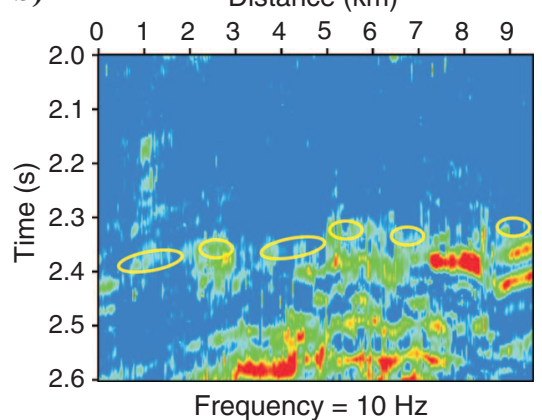

d)

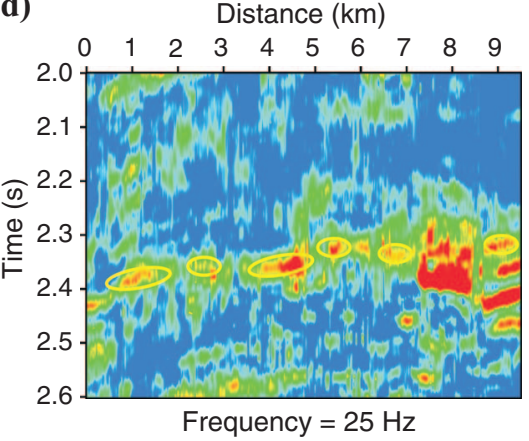

Figure 4. Multichannel matching pursuit for the time-frequency distribution. (a) Acrooked seismic line across six wells, indicated by vertical lines. (b-d) Three constant-frequency profiles at 10,20, and $25 \mathrm{~Hz}$. Circles indicate gas reservoirs. Underneath target gas reservoirs, the low-frequency shadows at $10 \mathrm{~Hz}$ are gradually reduced, and meanwhile the target gas reservoirs show strong amplitudes in 20 and $25 \mathrm{~Hz}$.

a)

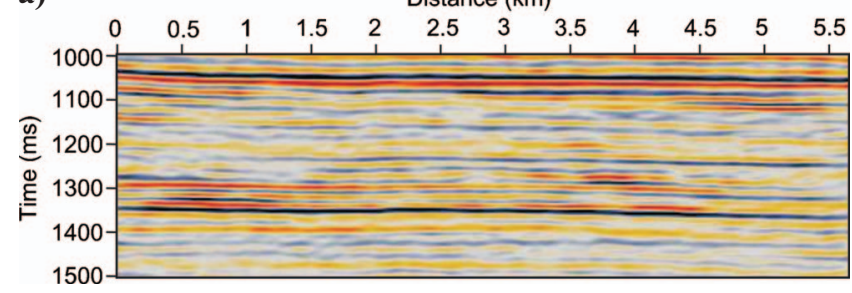

b)

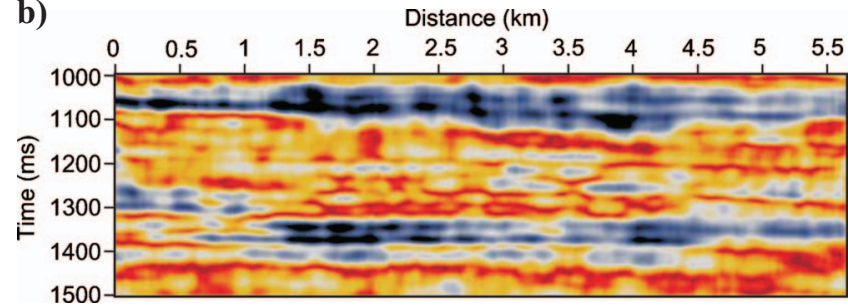

c)

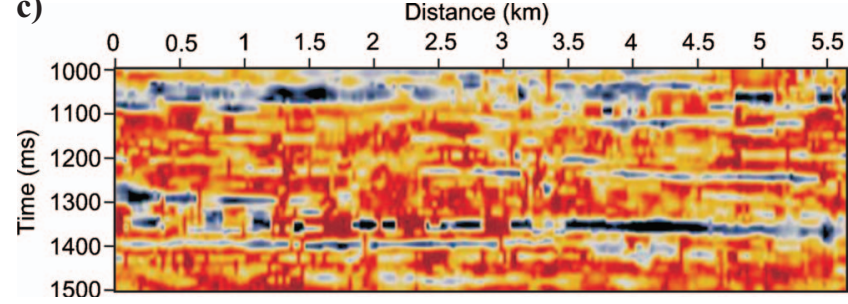

Figure 5. (a) A seismic section extracted from a 3D seismic cube. (b) The constant-frequency profile $(25 \mathrm{~Hz})$ generated by the waveform transform. (c) The constant-frequency profile $(25 \mathrm{~Hz})$ generated by the multichannel matching pursuit. time window, on which a nonstationary filter (accounting for the earth attenuation effect) could be attached. In the wavelet transform, the wavelet is fixed according to frequency and thus is different from the wavelet for convolution. This is also the case in the present version of matching pursuit decomposition.

Although the wavelet in a matching pursuit is adaptive to the data, it might be possible to exploit the wavelet concept in convolutional modeling and to generate another (soft) constraint in the time axis, in addition to the spatial axis, to improve further the uniqueness of matching pursuit decomposition. Ideally, if so, an atom or a basic wavelet $g_{\gamma_{n}}(t)$ from the matching pursuit could provide the wavelet for convolutional modeling, and the amplitude scale $a_{\ell, n}$ would be relevant to the reflectivity magnitude.

\section{CONCLUSIONS}

The technique of matching pursuit, with flexible wavelet size, is a powerful method to decompose a seismic trace into a series of wavelets. However, with single-channel matching pursuit, the solution is not unique and is also strongly affected by data noise. Multichannel implementation can improve the performance of matching pursuit decomposition, in which the lateral coherence between neighboring seismic traces is exploited as a constraint to overcome the nonuniqueness of the solution. An extracted wavelet should have an optimal correlation coefficient to a group of traces, instead of to a single trace only.

A robust implementation is based on linearity theory. According to linearity, an atom shared by neighboring traces is the best match to the average over multiple traces. Averaging might effectively suppress data noise and thus stabilize the procedure.

The MCMP scheme greatly improves spatial continuity in decomposition and temporal resolution in the resultant time-frequency spectrum. This spatial continuity is exploited to remove a strong coal-seam reflection from seismic data so as to allow the weak target reflections immediately on the top of the coal seam to be characterized. The MCMP scheme also can generate a plausible time-frequency spectrum for detecting low-frequency shadows underneath gas reservoirs.

\section{ACKNOWLEDGMENTS}

I am grateful to the sponsors of the Centre for Reservoir Geophysics, Imperial College London, for supporting this research. I also thank Charles Jones and Tim Sears for their thorough test on my previously published algorithm, which motivated me to pursue the research of this paper.

\section{REFERENCES}

Castagna, J. P., S. J. Sun, and R. W. Siegfried, 2003, Instantaneous spectral analysis - Detection of low-frequency shadows associated with hydrocarbons: The Leading Edge, 22, 120-127. 
Chakraborty, A., and D. Okaya, 1995, Frequency-time decomposition of seismic data using wavelet-based methods: Geophysics, 60, 1906-1916.

Durka, P. J., A. Matysiak, E. M. Montes, P. V. Sosa, and K. J. Blinowska, 2005, Multichannel matching pursuit and EEG inverse solutions: Journal of Neuroscience Methods, 148, 49-59.

Gabor, D., 1946, Theory of communication: Journal of the Institute of Electrical Engineers, 93, 429-457.

Korneev, V. A., G. M. Goloshubin, T. M. Daley, and D. B. Silin, 2004, Seismic low-frequency effects in monitoring fluid-saturated reservoirs: Geophysics, 69, 522-532.

Mallat, S. G., 2009, A wavelet tour of signal processing: The sparse way: Academic Press.

Mallat, S., and Z. Zhang, 1993, Matching pursuit with time-frequency dictionaries: IEEE Transactions on Signal Processing, 41, 3397-3415.
Morlet, J., G. Arens, E. Fourgeau, and D. Giard, 1982a, Wave propagation and sampling theory: Part I - Complex signal and scattering in multilayered media: Geophysics, 47, 203-221

, 1982b, Wave propagation and sampling theory: Part II — Sampling theory and complex waves: Geophysics, 47, 222-236.

Sinha, S., P. S. Routh, P. D. Anno, and J. P. Castagna, 2005, Spectral decomposition of seismic data with continuous-wavelet transform: Geophysics, 70, no. 6, P19-P25.

Studer, D., U. Hoffmann, and T. Koenig, 2006, From EEG dependency multichannel matching pursuit to sparse topographic EEG decomposition: Journal of Neuroscience Methods, 153, 261-275.

Wang, Y., 2007, Seismic time-frequency spectral decomposition by matching pursuit: Geophysics, 72, no. 1, V13-V20. 